\title{
An Inquiry into Female Dentists' Professional Lives and Concerns
}

\author{
Mona Rajeh"1,2, Richard Hovey', Shahrokh Esfandiari ${ }^{1}$ \\ ${ }^{1}$ Oral Health and Society Research Division, Faculty of Dentistry, McGill University, Montreal, Canada \\ ${ }^{2}$ Ministry of Higher Education, Riyadh, KSA \\ Email: shahrokh.esfandiari@mcgill.ca
}

Received 17 June 2014; revised 31 July 2014; accepted 15 August 2014

Copyright (C) 2014 by authors and Scientific Research Publishing Inc.

This work is licensed under the Creative Commons Attribution International License (CC BY).

http://creativecommons.org/licenses/by/4.0/

c) (i) Open Access

\begin{abstract}
Over the past 40 years, the number of female dentists has consistently increased. In this study, we intend to gain insight into the issues and concerns that influence the work characteristics of female dentists. Many studies have shown that gender differences exist in choice of specialization, practice patterns, and professional attitudes. We used a qualitative description methodology to guide this study, for which we recruited a purposive sample of six female dentists from the dental clinics of Montreal General Hospital, in the greater region of Montreal in Quebec, Canada. These dentists were general practitioners or specialists. Our findings suggest that female dentists consider the balance between work and childcare responsibilities challenging. For female dentists the relationship between work status and family happiness is an important matter to consider.
\end{abstract}

\section{Keywords}

Female Dentists, Professional Practice, Female Dentists Concern

\section{Introduction}

The proportion of women in dentistry has been consistently increasing over the past 40 years [1]. Some studies have shown that gender differences influence the choice of specialization, practice patterns, and professional attitudes [2] [3]; while others revealed that women favor primary care management practices rather than other dental specialties [4]. It is also suggested that women tend to work fewer hours, work part-time, and see fewer patients than men and are less likely to own their practices leading to a net income discrepancy between male and female dentists [2].

Female dental practitioners, much like the author, experience the dilemma of wanting to be perceived as competent dentists while fulfilling their role as a mother. Daily challenges to achieve professional goals and ful- 
fill marital and maternal obligations were driving force to conduct this research. It is ultimately critical to provide a supportive workplace for female dentists to provide high-quality services and to ensure that the productivity of the dental workforce is not negatively affected. This study intends to explore the issues and concerns that influence the professional practice for female dentists while attempting to reconcile familial and societal pressure. We also aim to describe the reasons that motivate the decisions made by these dentists regarding their dental practices and to document their voices and experiences.

\subsection{Gender and Feminization}

Recent studies examining gender heterogeneity among the health professions focusing on the stark contrast between males and females have shown that many health fields, including medicine and dentistry, are highly segregated by gender. This result was not exhibited in studies conducted during earlier periods [1]. In 2010, Adams performed a study examining the nature of this gender segregation in the healthcare professions in the United States and Canada for the periods of 1930-1931 and 2006-2008 [5]. The results showed that the percentage of female practitioners joining different health professions increased dramatically during these years, as many areas of expertise opened up. Reskin and Roos claimed that the increased number of women in the workforce was due to enhanced levels of education, social changes and occupational changes, as well as women's increased passion to practice within health professions and a concomitant steady or decreased enthusiasm among men [6]. Additional reasons for the feminization process may be the abundance of job opportunities in many health sectors [7]-[9] and the emigration of countless foreign female practitioners to developed countries such as the United States and Canada. As a result, many arguments have focused on whether the influx of women into traditionally male-dominated health occupations would lead to positive or negative consequences [1].

\subsection{Dental Services Provided}

There has also been a significant difference in the type of treatment provided by male and female dentists: periodontics, prosthodontics and implantology have been primarily dominated by male dentists, while female dentists have been pioneers in pedodontics, orthodontics and dental prophylaxis [3]. The dominance of men in high-status specialties, such as surgery, is not related to women having a lack of preference for such fields, but has rather occurred because a greater number of men enter this specialty [10]. Women's family responsibilities and the lack of female role models in dentistry may also be contributing factors. Therefore, it is important to better understand and foster awareness of the variables that could influence their career choices.

\subsection{Work Hours and the Practice Model}

Many of the available studies have provided evidence in support of a trend whereby women work fewer hours than men. Abate evaluated the variation between male and female dental practices in Ontario and observed substantial differences in working hours; females were more likely to work fewer hours than their male counterparts [11]. The average working hours per week for female dentists was 35.6, while the average working hours per week for male dentists was 39.5. Similarly, Aguila et al. also noted that female dentist, particularly those aged 25 - 40, work $10 \%$ fewer days, and treat $10 \%$ fewer patients than their male counterparts [2]. Female dentists also worked on a part-time basis more frequently than male dentists [12] [13].

The observed patterns of life-cycle labor-force participation of women, and the combination of work and household responsibilities, suggest that the relationship between work status and family happiness is of importance. Practice ownership is also linked to gender, as men are more likely to own their practice. Since qualifications and commitments to organizing a practice are commonly seen in men, they are often the primary earners in their families [14] [15]. With regard to self-employment, several studies have explained the multitude of factors that contribute to females' self-employment choices as opposed to those made by males, such as differences in male and female labor-market opportunities due to discrimination; experience and skill differentials; maternity and childcare concerns; and variations in lifetime occupational strategies [12] [16].

\subsection{Academia and Leadership}

In spite of the high inflow of females into the dentistry workforce, female dentists remain the minority among dental academics and leaders. Men with postgraduate qualifications hold most senior management positions, 
while women are more likely to be employed in faculty or government positions [14] [17]. Overall, the professionalization of dental practice has resulted in the continuation and consolidation of male dominance among specialists; studies highlight the fact that males still far outnumber females in terms of specialization in various fields [1] [18].

\subsection{Net Income}

A gender comparison in terms of net income among dentists showed that females earn less than their male co-workers, yet few articles have truly examined the differential between the wages of male and female dentists. Aguila et al. reported a significant earnings difference, finding that women's net income was $10 \%$ lower than that of men [2]. Brown and Lazar conducted a study to elucidate this matter by centering on female and male dentists who were similar with regard to their levels of professionalism and practice characteristics, and found female dentists have a mean net income 22\% below that of men [19]. Therefore, even if we equalize the level of education and practice pattern, gender will still play a part in preferential treatment in the dental market. These differences in income may be explained in that men tend to run their own dental business, whereas women generally work for others [1] [7].

In this study, we examined the personal and professional perceptions of female dental practitioners, as well as their goals and expectations. We believe that the findings of this study can lead to an improved understanding of women-related issues and inform policy makers and educators in order to make informed decisions about the societal role of female dentists and their work environment.

\section{Methodology}

\subsection{Design}

We chose a qualitative descriptive research design with the intention of describing the issues that influence the practice profiles of female dentists. A qualitative descriptive design is the methodology of choice when a researcher asks questions and seeks a straightforward and accurate description of a phenomenon [20]. Furthermore, qualitative research helps the researcher to gain a description of human experiences, such as those of female dentists. These qualitative studies can describe what already exists, determine the frequency with which something occurs, and categorize material to inform a specific topic of interest [21].

\subsection{Participants}

We recruited a purposive sample of six female dentists, who were either general practitioners or specialists in the greater region of Montreal in Quebec, Canada. We included female dentists who were general practitioners or specialists have at least two years of experience, 30-year-old and over, English-speaking and Montreal residents. We excluded female dentists who had less than two years of experience (to ensure that the dentists included have acquired sufficient experience to have encountered the concerns that could influence their practices); and any dental care professionals other than female dentists (e.g., dental students, dental assistants). The sample, composed of general practitioners and specialists, was unbalanced because we were interviewing very busy people who gave up their time to share their experiences.

After obtaining ethical approval from the Institutional Review Board of McGill University the participants were recruited from the dental clinics located in Montreal General Hospital. The associate dean of clinical affairs at the McGill faculty of dentistry introduced the researcher to a group of working female dentists. The researcher then contacted all participants via email to first request their participation. Although our sample size appears small, the sample size is aligned with the descriptive nature of the study design and sufficient to meet the rigors of an in-depth and detailed qualitative descriptive study [22] [23]. Smaller samples can be particularly relevant, when "rich" data is elicited from participants. Although the sample does not allow for generalizations, the details elicited from this methodology will shed new light for future research.

\subsection{Data Collection}

Semi-structured interviews with open-ended questions were the primary method of data collection. We used several key questions that acted as a guide to define certain areas that had to be explored; allowing the inter- 
viewer and interviewee to diverge from the topic to pursue an idea or response in more detail. Each participant was interviewed separately and privately in her private office or home. The participants were encouraged to talk freely about their experiences. The interviews lasted for approximately 60 to 90 minutes. However, the length and direction of each interview varied according to the participant's responses. The interviews were audio-recorded with the permission and written consent of the participants.

\subsection{Data Analysis}

Each interview was transcribed verbatim; a research assistant compared the written transcripts with the audiorecorded interviews to ensure accuracy. The transcripts were coded through qualitative content analysis. They were then read and reread until the research team gained a holistic understanding of the interview content. Key words and phrases were highlighted. Then each transcript was summarized and analyzed as a whole, and the large data set was condensed into smaller units of text. Coded categories emerged based on the highlighted words and labels. Categories and minor categories were grouped based on similarities and differences. Data was handled with utmost confidentiality and anonymity was secured during analysis.

\section{Results}

\subsection{Description of the Participants}

The participants consisted of two non-Canadian and four Canadian female dentists, whose ages ranged from 32 to 55 years, with a mean of 40.3 years. All but one of the six participants was married, and four had children living at home. Five of the participants were general practitioners, one was a specialist in orofacial pain, and one held a master's degree in dental science. The participants had two to 28 years of experience in dentistry, with approximately four reporting that they had at least 10 years' experience. Four worked full-time, and the other two were working part-time. Those who reported working full-time explained that they had to work part-time for some time, while on maternity leave, and mentioned being able to care for their child, including breastfeeding, as a significant consideration with regard to working part-time. Of the six participants, three indicated that they worked five days per week, two reported working four days per week, and one indicated that she works two days per week. Only two of the participants own their practice; of the remaining four, one reported owning her practice for four years, before giving it up to have children. Five of the participants stated that they work in a group practice. All six participants asserted that they selectively provided preventive management, operative treatments, simple endodontic and periodontal treatments, and implant restoration. None of the participants indicated that they provided surgical procedures or implant placements.

\subsection{Thematic Findings}

\subsubsection{Child-Care Responsibilities}

A major concern influencing women's dental practice pattern was identified to be child-care responsibilities. One participant explained that it was difficult for her to manage work and having children at the same time, explaining that she had to reassess her life priorities at the beginning of her career. Consequently, she decided to build her private practice first, which had its own challenges that were made more difficult because she had to take maternity leave for a few months, which was disruptive to her practice:

It was not easy at the beginning, but you have to set priorities, so at the beginning, I had my practice; I did a residency until 85, and I opened my private practice with somebody else. And it's not easy because, at the beginning, you have to stop sometimes for maternity leave.

It seems that the tension created by trying to balance work and childcare responsibilities affects women dentists because they believe it is women's place to raise their children, considering the stress of a full-time job and the added responsibilities for female dentists to deal with high-stress work and the demands of childcare obligations. From the above quotes, we can begin to understand that returning to work after maternity leave may have presented significant challenges for many female dentists. They seem to feel stressed about the extra tension created by their own perspectives, responsibilities and expectations between maintaining their professional lives and devoting themselves to full-time motherhood. They also seem to feel anxious about returning to their professional work and reluctant to leave their children after giving birth.

Many female dentists find it difficult to manage their professional lives and maintain their motherly roles. 
Childcare obligations are hard to combine with full-time practice. For these women it is important to overcome their daily struggle to balance between their work and their families. The following quote is from another participant who decided not to embark on her professional journey as a landlord dentist and to have children instead:

I was trying to figure out whether or not I should really venture out on my own or if I should stay an associate. Then I figured that it would be better to stay an associate while I wanted to have kids so that it wouldn't be so stressful.

Having children strongly affects female dentists' decision-making regarding the future of their professional careers. Given their stresses, many of these dentists sacrifice their careers to have children. Do female dentists feel more obliged to take care of their children than their partners? Is this in tune with socially accepted perceptions about gender roles within the family unit?

The pursuit of a balance between work and family appears to be a difficult equilibrium to attain, suggesting that we need to make this more explicit and open to discussion. This finding further suggests that there should be more flexibility in how female dentists negotiate their priorities between these two main vocations (i.e. work and children). Although female dentists have been striving to achieve the goal of a balanced life, they tend to have the luxury of choosing to be a mother rather than practicing dentist. This suggests that being a dentist is the only way for them to provide financial support for their children and families. On this matter, one participant said,

If you ask me which is the priority? I would say, being a mother is the priority and that being a dentist is really just a means for providing for my kids.

\subsubsection{Workplace Setting}

Another major category arising from the iterative process of content analysis was concerned with the workplace setting. The professional practice of many female dentists seems to be determined by the work setting characteristics of the dental office that they work in. Every dental office has its own practice standards, protocols, and culture. Dental offices and hospitals should meet appropriate and high standards of dental care to ensure the recruitment of a large number of highly qualified dental care professionals. However, one participant said:

While I was looking for my second and third part-time positions, because I did finally find other clinics that were more organized, that were better in following the protocols for infection control and treatment protocols for various types of dentistry.

The context of the workplace setting is a powerful tool to attract female dentists and encourage them to perform more effectively. Following standards and protocols is important not only to attract more professionals but also to prevent harmful dentistry.

I found it very disappointing because you think that once you get out of university that you are going to be heading toward this nice clinic that follows protocols and sometimes I saw things at that office that I found a little bit unnerving.

This participant's description of her experience while practicing dentistry in a private dental clinic supports the aforementioned claim that workplace strategy influences the practice profile of women dentists. Dental offices must be established with a primary goal to meet the needs of female dentists, to support and enhance their performance if they intend to retain this cohort of dentists.

Apparently, there is a relation between work conditions and the performance of women dentists. An unstable work environment appears to provide female dentists with fascinating challenges and concerns about their future. An unstable work environment might force these dentists to withdraw and start looking for other places to work. A participant recounted her concern by saying:

With everything that's happening with the MUHC (McGill University Hospital Center) now, my future was not so clear as far as where I would be able to practice, and so I felt now is a good time to at least take a step out the door to secure something for myself and [my] future.

This participant felt insecure and unstable, yet she decided to move her practice from a hospital setting to a private clinic in order to protect her future. Unstable work conditions seem to affect female dentists' satisfaction, interfere with their performance, and disrupt their career life options as well.

One participant gave an account of an unpleasant experience with her partner that negatively affected her practice and influenced her to sacrifice her private practice. The following quote justifies her choice:

Well, like I said, I had to sacrifice because I had a bad partner. If I didn't have a bad partnership, if my part- 
ner had helped me build it up to the point where it was running very smoothly, I would have kept the practice and I would have had my kids.

A collaborative relationship seems to be important to ensure the sharing of best practices and to provide highquality care to patients. Effective teamwork is necessary for the success of any dental practice more so for female dentists. The failure to implement effective teamwork and inter-professional collaborative relationships might result in inadvertent patient harm.

\subsubsection{Societal Obligation}

Social perspectives of responsibilities have also been identified as another reason that could influence the career decisions and preferences of female dentists. Unlike married ones, who are likely to make their schedules more family-friendly, unmarried female dentists tend to search for work that suits their needs and social/family commitments.

I picked a private practice that suited my schedule and my needs. One of the things, I didn't want to work weekends and minimal evenings. I just like having my evenings free for me for my social life.

Female dentists face multiple societal obligations. Given their chosen profession, they must find ways of reconciling their professional demands with their perceived personal obligations, which relate to their friends and themselves. Female dentists, however, may rise to the task. These women can be dentists while at the same time being friends, mothers, sisters, daughters, or wives. It is all a matter of choosing a line of work that fits their social obligations and way of life.

\subsubsection{Economics}

Dentistry being a health-related service, it is subjected to the highly volatile economic cycles that affect all sectors of society. For example, during times in which interest rates are high, female dentists will be more inclined to work more days per week and on a full-time basis. This will allow them to earn more money, save more, and generate more income via increased interest. This participant further noted:

It was a very difficult time because it was 1984, when there was a lot of unemployment, and the interest rate was $18 \%$, so it was very hard to set up a practice, I opened the office with a partner, but the interest rate was very high. So it was very hard for at least five years.

Conversely, during strained economic times, such as that experienced in 2009, during the height of the world's latest economic recession, dentists' net incomes decreased by more than 5\% [24]. Naturally, such a decline in income will affect how a female dentist manages her practice.

\subsubsection{Individual Preferences}

One final consideration has to do with the female dentists' individual preferences. Naturally, each practice profile will be different accounting for individual differences. A dentist's practice is directly affected by her needs, wants, preferences, and ideals. If a female dentist is to be successful in her practice, it must be set up in a manner that is consistent with who she is and what she wants.

Because I have a lot to do-you know, the other days I have other stuff to do-and I like it that way.

\section{Discussion}

The findings of this study indicate that family responsibilities, workplace settings, societal obligation, economics, and individual preference are all interwoven into the female dentists' practice profiles. The majority of our participants, however, were concerned with family responsibilities. This finding is consistent with a study conducted by Naidoo, which indicated that a woman's dual responsibility at home and at work was identified as a major factor influencing work patterns of female dentists in South Africa [25]. These health care providers are more likely to take on domestic responsibilities within households than their male counterparts when they start a family [26] [27].

Female dentists who have children are expected to deal with the responsibility of dedicated childcare. They are constantly trying to negotiate a balance between these two life domains to ensure that their productivity is not affected. Furthermore, failure to directly address the tension between professional and family life may cause adverse consequences such as stress and burnout syndrome [28]. It is suggested that one method of promoting the quality of a work environment-in favor of women dentists-is to encourage the use of frameworks and standards for developing "family-friendly” workplaces, which requires concrete policy approaches [29]. 
Participants in this study identified their workplace environment as one of the elements influencing their practice choices. They perform better when working conditions are adjusted to accommodate their specific needs during certain transitional stages (i.e., marriage/family) of their lives and careers. They need a professional working space, and they need to be able to set limits and determine acceptable behaviors at their places of work in order to be comfortable and professional. Similar findings were observed in a South African study where poor working conditions were strongly associated with job dissatisfaction [30]. Female dentists may choose not to practice to their fullest potential when they are dissatisfied by work conditions including their associates/partners at work. Ayers suggests that the work environment should motivate employees to perform at their best and show commitment to the organization, thereby enhancing work conditions to support the organization's mission and, thus, impacting on job satisfaction and work effectiveness [31]. Indeed, working conditions should meet the values and aspirations of female dentists and all health care providers.

Societal obligation may also influence female dentists' practice choices. Many of the challenges that face unmarried female dentists appear to be based on social attitudes. In many cases, if these professional single women were to give enough time to their careers, then it would mean sacrificing their social lives. In this way, we can say that female dentists experience hardship from the influence of social obligation and/or restriction. According to Risser et al., the time commitment and social compromises were the principal deterrents to entering the field of oral and maxillofacial surgery among female dental students in the United States [32].

Economics and financial matters were also issues that were raised by the participants. A female dentist may execute her career in dentistry within the standards of fulfilling her economic needs, and may develop the proficiency and clinical capability for independent broad practice, consisting of knowledge of health advancement and related obstacles. Fulfillment of economic requirements, particularly for female dentists, is an important element in the process of appraisal and evaluation of the types of the practices they perform. Decisions on what, where, when, and how to practice are influenced by numerous factors, including personal preferences [33]. These preferences were also identified as a key factor that helped influence career choices and workforce participation of doctors in their postgraduate years in Australia [34]. Female dentists may choose to practice parttime or only a few days a week as an individual choice, or they might decide to work part-time for various reasons, for example, to have free time in order to study or to simply to enjoy family life. They may decide to return to practice full-time when their children have grown older and are more independent, or when further education has been completed. Working full-time will bring focus to their careers and developmental growth. The crucial point is that female dentists should have the capacity to work when they are willing and able to do so.

\section{Conclusions}

We are witnessing a significant increase in the number of women choosing dentistry as a profession [35]. This correlates with the representation of women who apply to dental schools [36]. However, such changes provide new challenges and consequently necessitate an in-depth analysis and examination of the special concerns and needs for women at work. Could this be an artifact of the traditional model of the male- oriented dental curriculum at schools?

A supportive work environment helps encouraging women to utilize their skills and knowledge to the best of their ability. Women generally face a greater number of obstacles in order to succeed at work and female dentists are no exception. The societal norm that suggests the primary role of a woman is to fulfill family responsibilities may be to blame. Further studies are prompted to truly understand why female dentists choose different practice pattern than their male counterparts.

\section{Acknowledgements}

We wholeheartedly thank all of our participants for their valuable time and input into this research.

\section{References}

[1] Adams, T.L. (2005) Feminization of Professions: The Case of Women in Dentistry. Canadian Journal of Sociology, 30, 71-94. http://dx.doi.org/10.2307/4146158

[2] Aguila, M.A., Leggott, P.J., Robertson, P.B., Porterfield, D.L. and Felber, G.D. (2005) Practice Pattern among Male and Female General Dentists in a Washington State Population. American DentalAssociation, 136, 796-790. 
[3] Brennan, D.S., Spencer, A.J. and Szuster, F.S. (1992) Differences in Time Devoted to Practice by Male and Female Dentists. British Dental Journal, 172, 348-349. http://dx.doi.org/10.1038/sj.bdj.4807877

[4] Buddeberg, F.B., Klaghofer, R., Abel, T. and Buddeberg, C. (2006) Swiss Residents' Speciality Choices—Impact of Gender, Personality Traits, Career Motivation and Life Goals. BMC Health Services Research, 6, 137. http://dx.doi.org/10.1186/1472-6963-6-137

[5] Adams, T.L. (2010) Gender and Feminization in Health Care Professions. Department of Sociology, University of Western Ontario, London, 454-465.

[6] Reskin, B.F. and Roos, P.A. (1990) Job Queues, Gender Queues: Explaining Women’s Inroads into Male Occupations. Temple University Press, Philadelphia.

[7] Boulis, A.K. and Jacobs, J.A. (2008) The Changing Face of Medicine. ILR Press, Ithaca.

[8] Elston, M.A. (2009) Women and Medicine: The Future. Royal College of Physicians, London.

[9] Lindsay, S. (2005) The Feminization of the Physician Assistant Profession. Women and Health, 41, 37-61. http://dx.doi.org/10.1300/J013v41n04 03

[10] Gjerberg, E. (2002) Gender Similarities in Doctors' Preferences-and Gender Differences in Final Specializations. Social Science and Medicine, 54, 591-605. http://dx.doi.org/10.1016/S0277-9536(01)00054-5

[11] Abate, R. (2011) A Demographic Update for Ontario: Population and Dental Professionals. Ontario Dentists, 88, 3843.

[12] Ayers, K.M., Thomson, W.M., Rich, A.M. and Newton, J.T. (2008) Gender Differences in Dentists’ Working Practices and Job Satisfaction. Journal of Dentistry, 36, 343-350. http://dx.doi.org/10.1016/j.jdent.2008.01.012

[13] Riley, J.L., Gordan, V.V., Rouisse, K.M., McClelland, J. and Gilbert, G.H. (2011) Differences in Male and Female Dentists' Practice Patterns Regarding Diagnosis and Treatment of Dental Caries. Findings from the Dental PracticeBased Research Network. Journal of American Dental Association, 142, 429-440. http://dx.doi.org/10.14219/jada.archive.2011.0199

[14] Katrova, L.G. (2004) Gender Impact on the Socioprofessional Identification of Women Dentists in Bulgaria. Journal of Dental Education, 68, 19-22.

[15] Murray, J.J. (2001) Better Opportunities for Women Dentists: A Review of the Contribution of Women Dentists to the Workforce. British Dental Journal, 192, 191-196. http://dx.doi.org/10.1038/sj.bdj.4801333

[16] Gross, D. and Schäfer, G. (2011) “Feminization” in German Dentistry. Career Paths and Opportunities_A Gender Comparison. Women's Studies International Forum, 34, 130-139. http://dx.doi.org/10.1016/j.wsif.2010.12.003

[17] Atchison, K.A., Bibb, C.A., Lefever, K.H., Mito, R.S., Lin, S. and Engelhardt, R. (2002) Gender Differences in Career and Practice Patterns of PGD-Trained Dentists. Journal of Dental Education, 66, 1358-1367.

[18] Kaldenberg, D.O., Becker, B.W. and Zvonkovic, A. (1995) Work and Commitment among Young Professionals: A Study of Male and Female Dentists. Human Relations, 48, 1355-1378. http://dx.doi.org/10.1177/001872679504801106

[19] Brown, L.J. and Lazer, V. (1998) Differences in Net Incomes of Male and Female Owner General Practitioners. Journal of the American Dental Association, 129, 373-378. http://dx.doi.org/10.14219/jada.archive.1998.0215

[20] Sandelowski, M. (2000) Whatever Happened to Qualitative Description? Research in Nursing \& Health, 23, 334-340. http://dx.doi.org/10.1002/1098-240X(200008)23:4<334::AID-NUR9>3.0.CO;2-G

[21] Marriner, A. (1981) Research Design: Survey/Descriptive. Readings for Nursing Research. Mosby, St. Louis.

[22] Miles, M.B. and Huberman, A.M. (1994) Qualitative Data Analysis: An Expanded Sourcebook. 2nd Edition, Sage, Thousand Oaks.

[23] Patton, M. (1990) Qualitative Evaluation and Research Methods. Sage, London.

[24] Pollock, S. (2010) Economic Factors Affect Dental Trends. http://blog.go-iba.org/index.php/2010/05/21/economic-factors-affect-dental-trends/

[25] Naidoo, S. (2005) Women in Dentistry in South Africa: A Survey of Their Experiences and Opinions. Journal of Dental Association of South Africa, 60, 284-288.

[26] Ashri, N.Y., Norah, A.A., Mayyadah, A.M. and Rasha, A. (2009) Career Profile of Dentists in Saudi Arabia. Saudi Dental Journal, 21, 28-36.

[27] Smith, M.K. and Dundes, L. (2008) The Implications of Gender Stereotypes for the Dentist-Patient Relationship. Journal of Dental Education, 72, 562-570.

[28] Hansen, N., Sverke, M. and Näswal, K. (2009) Predicting Nurses Burnout from Demands and Resources in Three Acute Care Hospitals under Different Forms of Ownership: A Cross-Sectional Questionnaire Survey. International Journal of Nursing Studies, 46, 96-107. http://dx.doi.org/10.1016/j.ijnurstu.2008.08.002 
[29] Voss, E. (2009) Working Conditions and Social Dialogue-National Frameworks, Empirical Findings and Experience of Good Practice at Enterprise Level in Six European Countries (Draft Report). European Foundation for the Improvement of Living and Working Conditions.

http://www.eurofound.europa.eu/docs/events/confworkcond09/draftreport.pdf

[30] Kekana, H.P., Rand, E.A.D. and van Wyk, N.C. (2007) Job Satisfaction of Registered Nurses in a Community Hospital in the Limpopo Province in South Africa. Curationis, 30, 24-35. http://dx.doi.org/10.4102/curationis.v30i2.1068

[31] Ayers, K. (2005) Creating a Responsible Workplace. Human Resources Magazine. http://engagementisnotenough.com/pdfs/HR Magazine RBW.pdf

[32] Risser, M.J. and Laskin, D.M. (1996) Women in Oral and Maxillofacial Surgery: Factors Affecting Career Choices, Attitudes, and Practice Characteristics. Journal of Oral Maxillofacial Surgery, 54, 753-757. http://dx.doi.org/10.1016/S0278-2391(96)90697-7

[33] US Department of Health and Human Services (2008) The Physician Workforce: Projections and Research into Current Issues Affecting Supply and Demand. http://bhpr.hrsa.gov/healthworkforce/reports/physwfissues.pdf

[34] Australian Medical Workforce Advisory Committee (2002) Career Decision Making by Doctors in Their Postgraduate Years: A Literature Review. AMWAC Report 2002.1, Sydney.

[35] Jeanne, C.S. (2006) Global Health through Women’s Leadership: Introduction to the Conference Proceedings. Journal of Dental Education, 70, 5-7.

[36] Scarbecz, M. and Ross, J.A. (2002) Gender Differences in First-Year Dental Students' Motivation to Attend Dental School. Journal of Dental Education, 66, 952-961. 
Scientific Research Publishing (SCIRP) is one of the largest Open Access journal publishers. It is currently publishing more than 200 open access, online, peer-reviewed journals covering a wide range of academic disciplines. SCIRP serves the worldwide academic communities and contributes to the progress and application of science with its publication.

Other selected journals from SCIRP are listed as below. Submit your manuscript to us via either submit@scirp.org or Online Submission Portal.
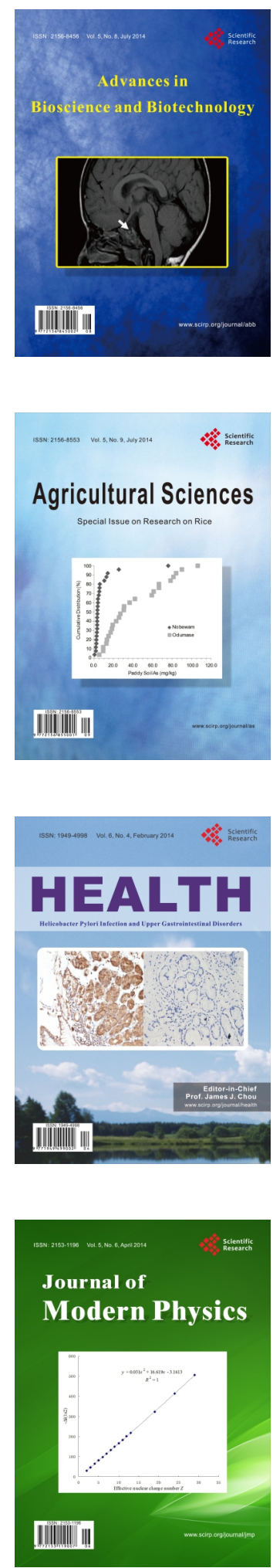
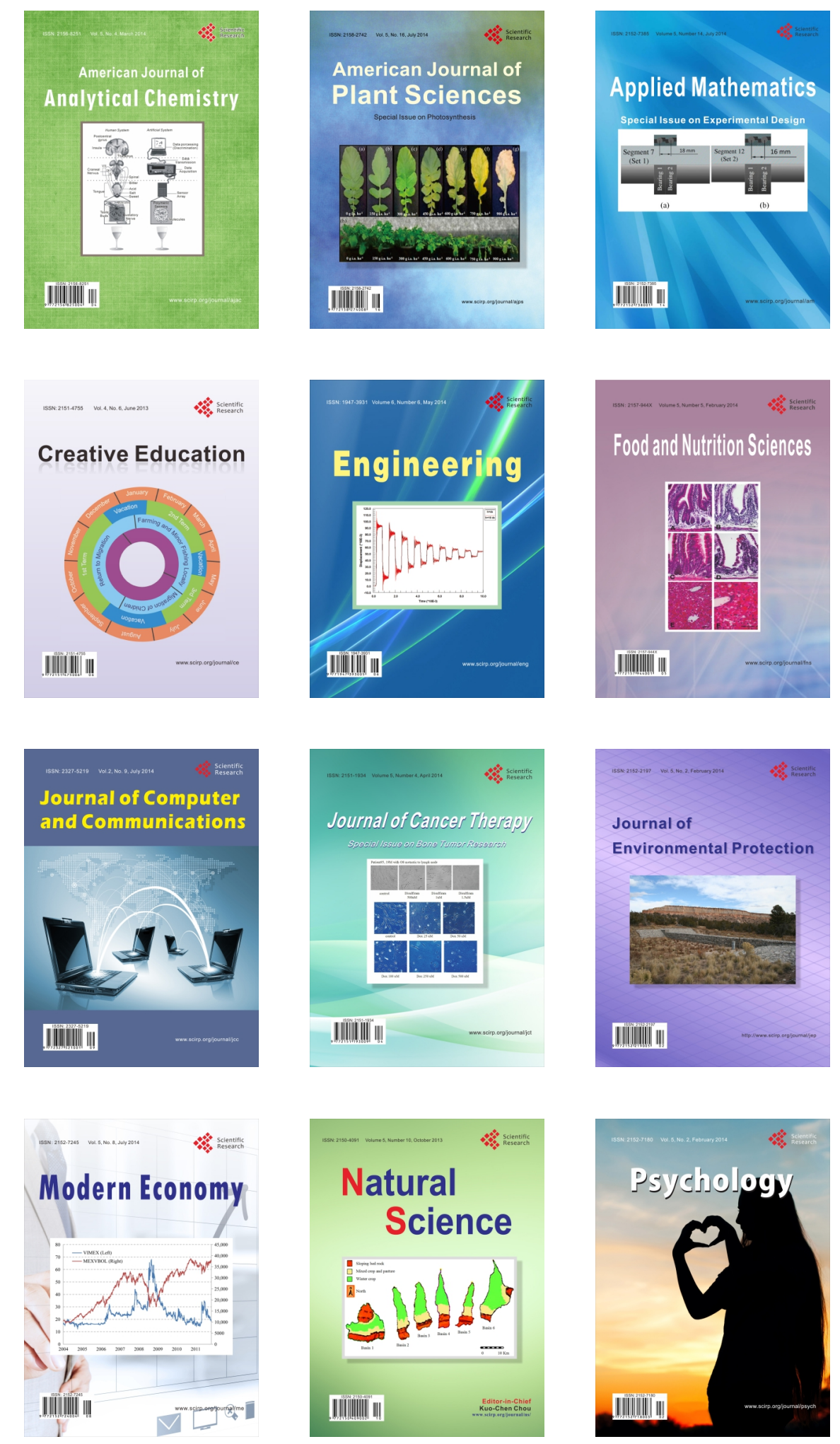